\title{
Erratum to: Multiple Aspects of Sexual Orientation: Prevalence and Sociodemographic Correlates in a New Zealand National Survey
}

\author{
J. Elisabeth Wells $\cdot$ Magnus A. McGee $\cdot$ Annette L. Beautrais
}

Published online: 21 April 2011

(C) Springer Science+Business Media, LLC 2011

\section{Erratum to: Arch Sex Behav (2011) 40:155-168 DOI 10.1007/s10508-010-9636-x}

We have discovered that the cumulative adverse event score was mistakenly calculated from the first four event types listed in Table 4 (beaten at home, other violence in the home, rape, sexual assault) and did not include the composite "Other adverse event." The odds ratios and the marginal percentage in the heterosexual group with no same-sex sexual experience, all produced from a model adjusting for age, sex, and age $\times$ sex, were correct for this revised definition of the cumulative adverse event score. However, the population percentages with each cumulative adverse event score were incorrect for this revised definition. The population percentage with each cumulative score from the four event types was $80.6 \%$ (SE 0.6) for no events, $12.9 \%$ (SE 0.5) for one event, $4.6 \%$ (SE 0.3) for two events, and $1.9 \%$ (SE 0.1) for three or four event types.

The online version of the original article can be found under doi:10.1007/s10508-010-9636-x.

\section{A. McGee}

Department of Public Health and General Practice,

University of Otago, Christchurch, Christchurch,

New Zealand

\author{
A. L. Beautrais \\ Department of Emergency Medicine, Yale University School \\ of Medicine, New Haven, CT, USA
}

\section{A. L. Beautrais}

Department of Psychological Medicine, University of Otago,

Christchurch, Christchurch, New Zealand

\section{J. E. Wells ( $\square)$}

Department of Public Health and General Practice,

University of Otago, Christchurch, PO Box 4345,

Christchurch 8140, New Zealand

e-mail: elisabeth.wells@otago.ac.nz 